\title{
Cheminots : des images et des représentations si prégnantes
}

Concluding remarks

\section{Christian Chevandier}

\section{OpenEdition}

\section{Journals}

Édition électronique

URL : https://journals.openedition.org/rhcf/175

DOI : $10.4000 /$ rhcf. 175

\section{Éditeur}

Rails \& histoire

\section{Édition imprimée}

Date de publication : 1 décembre 2007

Pagination : 366-414

ISSN : 0996-9403

Référence électronique

Christian Chevandier, "Cheminots : des images et des représentations si prégnantes », Revue d'histoire des chemins de fer [En ligne], 36-37 | 2007, mis en ligne le 10 mai 2011, consulté le 22 avril 2022. URL: http://journals.openedition.org/rhcf/175; DOI : https://doi.org/10.4000/rhcf.175 


\title{
Cheminots : des images et des représentations si prégnantes
}

\author{
Christian Chevandier \\ Maître de conférences en histoire contemporaine à l'université de \\ Paris I-Panthéon-Sorbonne
}

La commission «histoire sociale des transports par fer» de l'AHICF existe depuis 1995. Peut-être parce que la société ne peut s'appréhender que sur un temps long, elle n'a jamais travaillé à court terme. Ses trois grands chantiers ont tous pris du temps. Le plus important par ses résultats, qui permettent de connaitre le monde des travailleurs du rail mieux qu'aucun autre groupe professionnel, a été cette recherche coordonnée sur les origines sociales et géographiques des cheminots français, préparée pendant plusieurs années et pour laquelle ont travaillé ensemble des généalogistes cheminots, de jeunes étudiants et des chercheurs confirmés ${ }^{1}$. Les travaux menés sur la conflictualité, les mouvements sociaux, le syndicalisme ont aussi pris du temps. Le temps de l'entendement d'abord, puisqu'il a fallu longuement se demander quelle était cette combativité spécifique qui parait si robuste alors qu'une mise en perspective incite à être plus circonspect, à s'interroger sur la place des mouvements sociaux et des organisations du groupe social dans son identité, à se poser également la question des sources, sources connues, à inventorier, à inventer ou à construire qui nous permettraient de répondre à de telles questions. Cette besogne fut féconde et, en dehors même des journées tenues dans ce cadre ${ }^{2}$, un ouvrage sur l'histoire des grèves dans les chemins de $\mathrm{fer}^{3}$ et un dictionnaire biographique des militants cheminots ${ }^{4}$ peuvent être considérés comme ses productions. La confrontation des analyses, la mise en commun d'archives, la réflexion associant à propos d'un phénomène des chercheurs et des acteurs qui se connaissent bien peut apparaitre aujourd'hui comme exemplaire ${ }^{5}$.

1- "Origines sociales et géographiques des cheminots français », Revue d'bistoire des chemins de fer, $\mathbf{n}^{\circ} 22$ (printemps 2000).

2- «Les conflits sociaux dans les transports par fer», Revue d'bistoire des chemins de fer, n 19 (automne 1998).

3- Christian Chevandier, Cheminots en grève, ou la construction d'une identité (1848-2001), Paris, Maisonneuve et Larose, 2002.

4- Marie-Louise Goergen (dir.), Cheminots et militants. Un siècle de syndicalisme ferroviaire, Paris, Éditions de l'Atelier, 2003.

5- Je pense précisément aux séances consacrées à la grève de juin 1971 qui se sont tenues les 26 avril et 21 juin 2001 au Centre d'histoire sociale du XX siècle. 
L'activité dont le présent volume propose le produit est sans doute celle dont la durée a le plus surpris ; elle s'est prolongée plus d'une demi-décennie dans un monde où règne l'éphémère. Trois ans de séminaire trimestriel - « Les cheminots : images et représentations croisées », Paris, 2002-2005 -, un colloque de deux jours - « Images de cheminots. Entre représentations et identités ", Roubaix, 15-16 mars 2006 -, des discussions qui se prolongent à la séance suivante, des idées qui s'élaborent plus tard, la confrontation des textes définitifs aux prestations initiales témoignent de la vigueur de ce perfectionnement, des réponses enrichies des problématiques d'autrui ${ }^{6}$. Le temps long de la réflexion a joué là aussi, tout comme le suivi et l'aide des institutions qui ont porté ce projet. Je ne développerai pas plus longuement dans ces pages tout ce que l'on doit à l'AHICF, mais le Service du livre du Comité central d'entreprise de la $\mathrm{SNCF}^{7}$ et le Centre des archives du monde du travail, à Roubaix, ont aidé à cette confrontation entre l'historien et l'homme ${ }^{8}$ de terrain que Marc Bloch préconisait. La disponibilité, des années durant, des trois chercheurs qui ont coordonné cette entreprise et mis aussi la main à la pâte', leur complicité qui n'a jamais empêché de productives confrontations, ont été également des éléments de cette réussite. Je me permettrai enfin d'insister sur le fait qu'au séminaire comme au colloque un tiers des intervenants étaient des chercheurs du Centre d'histoire sociale du $\mathrm{XX}^{\mathrm{e}}$ siècle : il est peu commun qu'un laboratoire d'une telle taille s'investisse à ce point dans une initiative aussi pointue.

6- La dimension chronologique d'une réflexion commune a semblé si essentielle aux organisateurs qu'il a été décidé de ne pas reconstruire pour ce volume un plan qui aurait pu paraitre plus problématisé mais n'aurait pas tenu compte de l'agencement des rencontres et des échanges.

7- Les aspects matériels de ces manifestations ne sont pas secondaires. Tenues en début d'après-midi, ces séances étaient précédées d'un déjeuner pris en commun devant un magnifique paysage ferroviaire, dans un restaurant du comité d'entreprise de la SNCF, qui était souvent l'occasion de revenir sur la séance précédente ou d'aborder, déjà, le thème de celle de l'après-midi.

8- En ce début de XXI ${ }^{\mathrm{e}}$ siècle, nous soulignerions que l'homme de terrain peut être une femme.

9- Les textes de Marie-Suzanne Vergeade et Georges Ribeill se trouvent dans ce volume. Marie-Suzanne Vergeade a écrit «Images de cheminots, XIXe-XX siècles » et je suis l'auteur de «La fresque, la ville, les cheminots» dans le $\mathbf{n}^{\circ} 22$ (juin 2007) de Histoire et sociétés. Revue européenne d'histoire sociale qui consacre un dossier aux images des cheminots. 
Je ne vais pas résumer ici les contributions des pages précédentes dont le lecteur peut prendre connaissance et dont il verra ainsi à quel point elles alimentent les lignes qui suivent, mais tenter de les mettre en perspective, d'avancer des hypothèses, de développer des points significatifs tout en ne perdant pas de vue qu'une identité se bâtit sur une multitude d'héritages, les spécificités de l'entreprise, les pratiques des métiers, les bases juridiques et réglementaires qui peu ou prou façonnent le groupe, l'adéquation du marché du travail aux horizons économiques, sociaux, politiques des activités. De cet ensemble, images et représentations des cheminots, qui existent bien avant les années 1890, lorsque le mot est adopté avec cette acception (et cette orthographe), ne sont qu'un élément, et ce n'est pas parce que notre regard s'y porte qu'il faut ne voir que lui.

\section{Images de cheminots : saisir des hommes, saisir un groupe}

Les images de cheminots sont multiples : croquis, dessins, peintures, photographies, films documentaires ou de fiction, documents argentiques, magnétiques, numériques. Et, par delà l'image, combien d'œuvres utilisent un registre ferroviaire pour aborder des thèmes qui l'intègrent, comme la sculpture de Boris Zaborov ${ }^{10}$ qui place dans un grand bronze le livre d'homologation des tarifs des chemins de fer de la chambre de commerce de Paris ${ }^{11}$ ? Pas d'approche de l'image et de la création en général sans recours à une intertextualité qui va au-delà des textes. Les films savent aussi se copier sans se paraphraser, et le spectateur de Bataille du rail peut bien penser à la scène de la bataille de The Generall'2, lorsque la locomotive détruit un pont et tombe dans un cours d'eau. Les cheminots ont pourtant repris à leur compte la fameuse scène des sirènes au moment de l'exécution, instant le plus intense du film que René Clément avait créé pour sa dramaturgie ${ }^{13}$ et qui est

10- Philippe Bidaine et Pascal Bonafoux, Boris Zaborov, Milan, Skira, 2007.

11- Composition avec poupées, 1996

12- Buster Keaton, 1927.

13- Le tournage de La Belle et la Bête, dont René Clément était co-réalisateur, s'est déroulé presque en même temps que celui de Bataille du rail. Alors que Clément lui parlait de cette scène intense mais sortie de son imagination, Cocteau assura qu'elle allait rapidement acquérir un statut d'authenticité et rappela la rencontre en sa présence, une dizaine d'années après la sortie du film et plus de trente ans après les faits, entre S. M. Eisenstein et un ancien marin du Potemkine qui, expliquait-il, s'était trouvé « sous la bâche » au moment initial de la mutinerie. Tous deux se congratulèrent avec beaucoup d'émotion et le réalisateur soviétique reprit son chemin avant de se rappeler qu'il avait inventé la scène de la bâche; Philippe Esnault, entretiens avec René Clément, «À voix nue », France-Culture, 1995. 
passé dans la légende ${ }^{14}$. Ainsi, si l'image façonne l'identité des cheminots, elle ne joue aucun rôle sur sa pratique, sauf par le biais normatif des films d'entreprise à finalité formative étudiés par Michel Ionascu ${ }^{15}$. En cela la différente est forte avec d'autres groupes sociaux, ceux dont les moments les plus extrêmes constituent le clou de films de fiction destinés au grand public. C'est de longue date le cas des policiers ${ }^{16}$, mais les séries télévisées l'étendent désormais à d'autres groupes, les pompiers, le personnel hospitalier, etc.

La dimension technique est constitutive du métier de cheminot. La technique de la machine à vapeur, celles de la traction thermique, de la traction électrique, de la voie ferrée, la technologie ferroviaire sont essentielles dans ces représentations. Il est une autre technique qui a façonné l'image des travailleurs du rail, qui apparait avec le chemin de fer et se développe lorsque ses employés deviennent cheminots, c'est la photographie, quitte à ce que le cliché se transforme en dessin pour fossiliser l'image du cheminot gréviste ${ }^{17}$. Le corpus considérable accumulé par Henri M. Petiet ${ }^{18}$ est significatif de cet engouement. Fils d'une famille d’ingénieurs de la Compagnie du Nord, ce garçon né en 1894

14- En revanche, certaines scènes du film, sans être directement inspirées par des faits précis, ont pu correspondre à des actions accomplies au cours de l'été 1944. Celle du mécanicien retraité sollicité par les résistants (« N’y va pas, Jules, c'est tous des fous ! ») évoque un épisode de la Libération de Paris, celui du train allemand bloqué sous le tunnel de Ménilmontant : «Le commandant Darcourt dit qu'il fallait décrocher la locomotive pour éviter toute tentative de sortie du train. Mais pour cela, il fallait un cheminot. Quelqu'un eut l'idée d'aller chercher un retraité de la SNCF qui habitait dans les HLM. Cet homme âgé était en train de faire la vaisselle, il écouta attentivement ce qu'on lui disait, s'essuya les mains et descendit sur le terrain. Il parvint à se glisser sous la locomotive pour la décrocher puis la faire avancer de deux cents mètres. Dès lors, les Allemands, bloqués dans les wagons, se rendirent », Madeleine Riffaud, On l'appelait Rainer, Paris, Julliard, 1994, p. 139.

15- Michel Ionascu, «Cheminots argentiques : l'image d'un groupe social dans le cinéma et l'audiovisuel français », thèse de recherches cinématographiques et audiovisuelles, université de Paris III, 1999, p. 107-110.

16- Pour un exemple de cet effet en opération, Bénédicte Desforges, Flic. Chronique de la police ordinaire, Paris, Michalon, 2007, p. 185. Des courts-métrages normatifs, opposant «l'intervention correcte et l'intervention maladroite» ont été tournés et diffusés à fin de formation aux gardiens de la paix de la préfecture de police de Paris au début du XX siècle, Jean-Marc Berlière, Le Monde des polices en France, Bruxelles, Complexe, 1996, p. 75.

17- Le Petit journal, 1910.

18- Ses 37500 cartes postales et 9100 tirages ont été vendu à l'Hôtel Drouot le 26 novembre 2004 . 
avait commencé très tôt une collection de modèles réduits, d'ouvrages de toutes sortes. Les clichés de sa collection représentent surtout des machines, des convois, mais ne fixent qu'exceptionnellement des cheminots. Encore est-ce généralement dans des conditions exceptionnelles, comme ces photographies de grèves auxquelles nous sommes accoutumés ${ }^{19}$. Il n'en reste pas moins qu'une de ses plus belles pièces, «Le repas des cheminots » reproduit ici p. 50, montre un groupe de trois employés de la Compagnie de l'Est du début du siècle qui ont installé leur table sur une voie. Mais la vraie vedette, que l'on voit en premier, celle qui occupe la moitié du cliché (alors que le groupe n'en prend que le quart), est une magnifique locomotive à l'impressionnante cheminée.

La photographie, montrerait-elle un phénomène exceptionnel, est un discours ; par elle-même elle se fait truisme, veut attester une généralité. Alors que les dirigeants de la fédération CGT des cheminots avaient fait en 1936 ce qu'il fallait pour que les travailleurs du rail ne se lancent pas dans le conflit social ${ }^{20}$, c'est une photographie « des cheminots grévistes d'Anzin, le 12 juin 1936 » qui illustre la couverture de l'invitation de l'Institut [confédéral] d'histoire sociale de la CGT à la journée qui, soixante-dix ans plus tard, célèbre cette année-là. Ce cliché de travailleurs d'un groupe à la pointe des luttes de la fin du $\mathrm{XX}^{\mathrm{e}}$ siècle ${ }^{21}$ est bien une photographie du Front populaire, groupe d'hommes d'un

19- Rémy Cazals, "L'événement dans la cité : la grève ", in Alain Boscus et Rémy Cazals, Sur les pas de Jaurès. La France de 1910, Toulouse, Privat, 2004 p. 193-212, et Madeleine Rébérioux, «La carte postale de grève : propos sur une collecte et une exposition », Le Mouvement social, $\mathrm{n}^{\circ} 131$ (avril-juin 1985), p. 131-144.

20- C'est précisément l'exemple que prend Antoine Prost pour démontrer la volonté du parti communiste, très influent dans la fédération, de ne pas étendre les grèves, « Le PC et Léon Blum face aux grèves de 1936 », Sciences humaines, "L'histoire aujourd'hui », hors série $\mathbf{n}^{\circ} 18,1997$, p. 11.

21- C'est bien cette identification, aujourd'hui, des cheminots à la pratique gréviste qui joue ici. Elle est commune, a déjà été relevée à de nombreuses reprises, et persiste, se manifeste comme dans ce lapsus calami d'un syndicaliste postier qui écrit dans le journal de l'Institut d'histoire sociale CGT-PTT : «En 1963, de Gaulle avait dû remballer "sa réquisition" face à l'ampleur de la grève des cheminots » (Le Relais, $\mathbf{n}^{\circ} 34$, juin 2007). Il ne s'agit pas d'une erreur, les syndicalistes de la génération de l'auteur de l'article se souviennent fort bien de la grève des mineurs et les postiers, par la place qu'avaient alors les centres de tri, étaient très sensibles aux mouvements sociaux dans les chemins de fer ; il n'en est que plus significatif que le mot «cheminots » s'impose ainsi pour suivre celui de " grève ». Notons par ailleurs que, deux ans plus tôt, après une journée de grève pour une augmentation salariale suivie par 300000 cheminots, en mai 1961, le travail avait repris et le gouvernement avait pris un décret de réquisition, le dernier à ce jour contre les cheminots. Lors de la grève de Pâques 1962, très suivie, le nouveau Premier ministre, Georges Pompidou, avait eu la prudence de s'abstenir. 
âge mûr ${ }^{22}$, visage sérieux et poing levé, en rien une photographie de cheminots : pas le moindre signe ferroviaire comme cette grosse locomotive en carton de la manif parisienne du $1^{\text {er }}$ Mai de l'année suivante ; même la baraque, dans le fond, renvoie d'abord au paysage de l'usine ${ }^{23}$. Mais cette absence s'inscrit dans un continuum. Les agents des chemins de fer sont rares sur les toiles. Celle du peintre américain Albert Herter, immense (plus de $60 \mathrm{~m}^{2}$ ), Le Départ des poilus, août 1914, exposée dans la halle Alsace de la gare de l'Est où elle fut inaugurée en 1926, ne présente pas le moindre cheminot. Même les tableaux de Maximilien Luce, qui représentent des groupes de permissionnaires dans les « Gares de Paris pendant la guerre $»^{24}$, ne montrent aucun cheminot ${ }^{25}$. Ces gares guerrières ne sont d'ailleurs pas une exclusivité de Luce : Dans le halle de la gare de l'Est, peint/dessiné en 1916 par Théophile Steinlen ${ }^{26}$, ne présente lui aussi pas le moindre travailleur du rail ${ }^{27}$. Steinlen, dessinateur du mouvement socialiste et figure du Montmartre du tournant du siècle, était comme Luce habitué à dessiner des travailleurs. Quelle énigme que cette défection lorsque les ouvriers de la métallurgie étaient communément peints ou sculptés ! Est-ce que les machines, les machines de Claude Monet à Saint-Lazare et celles de Raoul-André Ulmann à Bercy, cachent ceux qui les conduisent, ceux qui les servent, ceux qui les entretiennent ? Pourquoi alors peuvent-ils apparaitre en d'autres pays, comme sur les toiles d'Hans Baluschek ${ }^{28}$ ? Quant au garde-barrière barbu

22- Il n'y avait pas eu de recrutement dans les chemins de fer depuis le début de la crise. 23- Mais le discours est ici est volontaire et ne relève pas de l'ignorance comme ce fut le cas de ce sujet sur la grève de 1936 dans les chemins de fer proposé par l'Éducation nationale aux collégiens qui se présentaient au brevet en 1997 dans les académies de Besançon, Lyon, Nancy-Metz, Strasbourg et Reims.

24- Il s'agit du titre de l'exposition de ces œuvres qui s'est tenue du 23 octobre au 16 novembre 1916 chez Bernheim-Jeune, Philippe Cazeau, Maximilien Luce, Lausanne/ Paris, La Bibliothèque des Arts, 1982, p. 167-169.

25- Voir les tableaux reproduits dans Maximilien Luce. Peindre la condition humaine, Paris, Somogy-Editions d'art, 2000, p. 52-57, qui, à l'exception des Escaliers de la rue d'Alsace (1916, musée de l'Hôtel-Dieu, Mantes-la-Jolie), font partie de collections particulières.

26- Genève, musée du Petit Palais.

27- Mais peut-être les cheminots n'ont-ils pas vraiment leur place, même dans une gare, en de telles circonstances ? Voir, à l'issue de la guerre suivante, les photographies du Retour des prisonniers faites sur une commande de la SNCF en 1945 par Willy Ronis.

28- Plusieurs de ses œuvres mettent en scène des travailleurs des chemins de fer, notamment la toile (peinte en 1928) qui représente une famille : la garde-barrière, le cantonnier et leur fille, An der Strecke, Musée Bröhan de Charlottenburg. Sur ce peintre, Margrit Bröhan, Hans Balushek 1870-1935. Maler-Zeicher-Illustratör, Berlin, Bröhan Museum, 2002. 
du tableau de Laurits Andersen Ring ${ }^{29}$, il en est le principal personnage, bien loin de ces silhouettes esquissées en cette même fin du XX $\mathrm{XX}^{\mathrm{e}}$ siècle par les peintres français.

Rares sur les toiles, rares sur les photographies. La revue Correspondances, à laquelle ont collaboré plusieurs auteurs de textes du présent recueil, est richement illustrée. Sur les 71 photographies représentant une scène ou un paysage ferroviaire de son numéro de janvier 2007, il n'y en a que sept où l'on peut voir un homme ou une femme, toujours minuscule, parfois le mécanicien dans la cabine. Une approche systématique et comparative nous instruirait plus précisément, mais il semble que la photographie ferroviaire n'est qu'exceptionnellement une photographie de cheminots. Est-ce le cas uniquement en France ? Puisque nous devons à un universitaire du Wisconsin un des textes de ce volume, feuilletons le calendrier 2007 de la Milwaukee Road Historical Association : pour les 13 grandes photographies, on peut voir des voyageurs ou des passants sur trois d'entre elles, des cheminots (discrets) sur deux et les autres, plus nombreuses, ne révèlent aucune présence humaine $^{30}$. Mais si nous étudions le calendrier de 2004 De Paris à la Méditerranée $e^{31}$ de Loco Revue (treize photographies), une comprend un groupe d'hommes indéterminés, voyageurs ou/et cheminots, six des cheminots, dont les agents qui ont pavoisé la 4138 à Modane pour le 14 juillet 1898, mais aussi des travailleurs de la voie sur des poteaux télégraphiques, parfois simplement le mécanicien ou le chauffeur dans la cabine : rares, petits, mais pas absents. Pourtant, quelles que soient les raisons juridiques qui dissuadent de prendre des clichés d'hommes et de femmes, ils sont bien là ${ }^{32}$.

29- Banvakten (1884), Nationalmuseum, Stockholm.

30- Sur une ligne de la Milwankee Road, une photographie prise en 1935 de l'Atlantic 221 profilée du Hiawatha est construite autour du conducteur, situé légèrement audessous du point central de l'image, La Vie du rail, Hors série «Les 100 plus belles locomotives ", avril 1996, p. 36-37.

31- Keith Mann estime que le PLM est la compagnie qui évoque le plus sûrement la Milwankee Road.

32- J'ai en septembre 2003 pris 180 photographies aux Ateliers SNCF d'Oullins; une seule d'entre elles laisse voir des hommes (préoccupés la partie de boules à laquelle ils se livrent). Le site était pourtant ce jour-là en activité. C'est bien sûr le choix du photographe qui a joué : je n'ai pas voulu demander systématiquement l'autorisation de photographier. 
Images de cheminots, mais aussi, maintenant, image de cheminotes. La question du genre a été à de nombreuses reprises abordée dans les textes précédents, et sans doute, là aussi, conviendrait-il de voir ce qui se passe ailleurs. Quelle représentation des femmes qui exercent le métier de pompier, de policier ${ }^{33}$, tous ces métiers où prévalait et prévaut encore une culture masculine, voire machiste, mais aussi quelle représentation des hommes qui exercent dans des métiers considérés comme féminins? Les infirmiers s'accommodent avec le passé des hôpitaux d'avant qu'on y soit qualifié, mais aussi avec les à-côtés, les infirmiers psychiatriques qui jusqu'à la camisole chimique étaient des hommes, les infirmiers militaires. Qu'en est-il alors des rarissimes maieuticiens, puisque le terme "sage-homme» a été délaissé, des puériculteurs? Les cheminotes, le sens même en a changé puisque, dans l'entre-deux-guerres, la cheminote était la femme du cheminot, comme la colonelle ou la générale était l'épouse d'un officier, et que « faire la cheminote » signifiait ne pas travailler après le mariage, rester à la maison et s'occuper du foyer ${ }^{34}$. Les femmes n'ont pourtant pas été absentes du monde des travailleurs des chemins de fer ${ }^{35}$. Les gardes-barrière ne sont en rien un contre-exemple : dès le début du XX $\mathrm{XX}^{e}$ siècle, des femmes ont pu travailler dans certains ateliers, quitte à ce que ce soit pour des activités considérées comme « féminines », la sellerie, le garnissage, le bobinage même, alors que, avec l'électrification de la traction, les bobines ont acquis une tout autre dimension. Dans les services administratifs, elles étaient bien présentes. C'est sans doute dans les directions que l'on croisait le plus de femmes employées des chemins de fer, à tel point que des toilettes distinctes y étaient aménagées ${ }^{36}$. Il n'empêche que les images de cheminotes sont encore rares; il s'agit d'une des raisons pour lesquelles le Comité d'établissement régional SNCF Nord - Pasde-Calais a édité pour l'année 2007 un calendrier où chaque mois est

33- Valérie Broussard, Marc Loriol et Sandrine Carolie, « Une féminisation sur fond de segmentation professionnelle genrée : le cas des policières en commissariat ", Sociologie pratique, $\mathrm{n}^{\circ} 14$ (2007), p. 75-88.

34- Jean-Louis Burdy, Le Soleil noir. Un quartier de Saint-Étienne (1840-1940), Lyon, Presses universitaires de Lyon, 1989, p. 136. Cette expression se comprend mieux à la lecture des pages sur l'activité et la sociabilité féminine, p. 33-38, 133-153 et 155-176.

35- Sans doute l'histoire sociale trouverait-elle en ce groupe féminin atypique un sujet de choix.

36- Les toilettes femmes d'un des services de la direction de la SNCF étaient ainsi d'une jolie couleur rose tandis que celle le carrelage de celles de leurs collègues masculins étaient bien sûr bleu ciel. 
illustré par le dessin d'un auteur de bande dessinée sur ce thème. Pour le mois de janvier, Carali a croqué une chauffeuse qui alimente la chaudière d'une loco à vapeur sous le regard frigorifié d'un bovin.

Après le séminaire, la nécessité est apparue d'élargir notre approche. Il fallait regarder ailleurs, aller voir d'autres groupes pour être à même de voir nos cheminots. Un matériau de premier choix s'offre pour une telle démarche qui ne serait efficiente que comparative. Plus de 4000 photographies sur le travail et les travailleurs ont été prises par François Kollar entre 1931 et 1934 pour une enquête des éditions "Horizons de France ». Fils d'un cheminot et ayant lui-même travaillé dans les chemins de fer tchèques, ancien tourneur chez Renault, ce photographe d'origine hongroise né en $1904^{37}$ a choisi parmi ces milliers de clichés la matière de 15 fascicules thématiques de la série La France travaille, toujours accompagnés d'un texte d'une forte tenue. Le Railis associe l'œuvre picturale de Kollar et celle, écrite, de Pierre Hamp (tout comme Mineurs et Les Métiers du fer). Si sa couverture présente deux cheminots au travail à la proue d'une Pacific de l'État, tout comme Mineurs propose un travailleur des houillères, le volume consacré au textile montre des machines, mais personne pour les actionner. Ce corpus considérable, qui mérite bien sûr d'être étudié en fonction des outils classiques de l'historien, pourrait sans doute permettre de répondre à nos questions.

Parmi les travailleurs du rail, hors des mécaniciens dans la cabine de leur loco $^{39}$, il est un groupe qui a été beaucoup photographié, celui des cheminots en usine, ces ouvriers d'Ateliers qui ont posé, immobiles, face au photographe. Mais qu'il s'agisse de photographies de groupe, tous ensemble comme dans les cours d'école ${ }^{40}$, ou de celles où l'on se

37- Après sa mort, en 1979, deux expositions ont présenté son œuvre à Paris, en 1985 à l'Hôtel de Senlis et en 1989 au Palais de Tokyo. En 2004, certains de ses clichés et de ceux de Lee Friedlander ont été présentés au Palais de Sully pour l'exposition $A t$ Work. 38- En 1986, sous le titre Cheminots des années 30. Les hommes de l'État, les éditions La Vie du rail ont publié les photographies de François Kollar conservées dans la collection de la bibliothèque Forney. La même année, un ouvrage présente l'ensemble de l'œuvre : Anne-Claude Lelieur et Raymond Bachollet, La France travaille. Regard sur le monde du travail à la veille du Front populaire, Paris, Le Chêne.

39- Rarissimes se révèlent les photographies d'autres travailleurs des chemins de fer, comme celle du "contrôleur de train» dans la série Les Serviteurs anonymes de Jean Michaud (vers 1952), BnF, Estampes et Photographies, Ep1517Fol.

40- C'est le cas de la photographie des ouvriers de l'Atelier du modelage prise en 1881 aux Ateliers PLM d'Oullins, fonds de la bibliothèque municipale de Lyon. 
fige dans la posture du travail ${ }^{41}$, ces clichés sont bien ceux de la France industrielle, des gens des ateliers et des usines ${ }^{42}$. Il faut attendre le début du XXI ${ }^{\mathrm{e}}$ siècle pour que, à l'occasion de l'exposition « Mémoires de la vie du rail », une série de clichés des anciens ouvriers d'un Atelier SNCF désaffecté soit effectuée par Cesare de Librio ${ }^{43}$. Les photographies en noir et blanc du site ferroviaire vide y alternent avec des portraits de retraités, qui pour certains tiennent des objets du travail, et quelques portraits de groupe. Impression étrange que ces figures en noir et blanc, sur fond noir, d'hommes qui ne sont plus des cheminots, mais le sont tant encore, accentuée par la conscience d'un cadrage de paysages dans lesquels on ne peut percevoir la reconversion en cours, soulignée par une muséographie dépouillée. C'est bien la fécondité du monde, du monde cheminot qui se montre ainsi, hors de toute démarche de deuil alors que l'on est si près des Alyscamps dont la construction des Ateliers du PLM semblait augurer la disparition. Mais ces hommes sont représentés alors que, quand bien même les bâtiments subsistent, leur vocation ferroviaire n'est plus, un peu comme des miraculés qui n'ont pas sombré avec les Ateliers. Cette démarche est d'une toute autre nature que celle qui se trouve à l'origine du petit monument qui, à l'entrée des ateliers ferroviaires de Stendal, en Saxe, accueille les visiteurs : sur une stèle, trois médaillons représentent un wagon, une locomotive et, entre eux, le sujet central : un forgeron ${ }^{44}$ (fig. 1).

41- Archives départementales de la Dordogne, Hommes et Ateliers du PO, Périgueux, Conseil général de la Dordogne, 1997. Présenté sous une forme originale, il s'agit d'un double album, avec d'un côté du livre des photographies prises entre les années $60 \mathrm{du}$ XIX siècle et celles du xxe siècle, et de l'autre un corpus de clichés récents.

42- Denis Woronoff, La France industrielle. Gens des ateliers et des usines 1890-1950, Paris, Le Chêne, 2003. Voir ainsi la photographie de l'atelier de montage des locomotives au Creusot, p. 114-115. La disjonction dans la deuxième moitié du XIX siècle entre les fonctions de production de matériel ferroviaire, confiées à d'autres entreprises, et celle de réparation, généralement conservées par les compagnies de chemin de fer, donne l'impression d'activités plus différentes qu'elles ne l'étaient en réalité.

43- Cesare De Librio, Mémoires de la vie du rail, Arles Musée Réattu, 2006 ; Virginie Buisson, «Traverses et ballast », p. 10-15. L'exposition s'est tenue à l'espace Van Gogh du 10 décembre 2006 au 29 janvier 2007. Les œuvres ont été acquises par le musée Réattu pour sa collection photographique.

44- Une histoire de ce monument qui a traversé des régimes (le Reich bismarckien, la République de Weimar, la dictature hitlérienne, l'occupation soviétique puis le régime se réclamant du socialisme de la République démocratique allemande), qui tous ont manifesté un intérêt particulier au monde du travail, serait passionnante. À proximité d'une autre entrée, un ajustement inhabituel de briques signale l'emplacement de croix gammées enlevées à la fin de la guerre. 


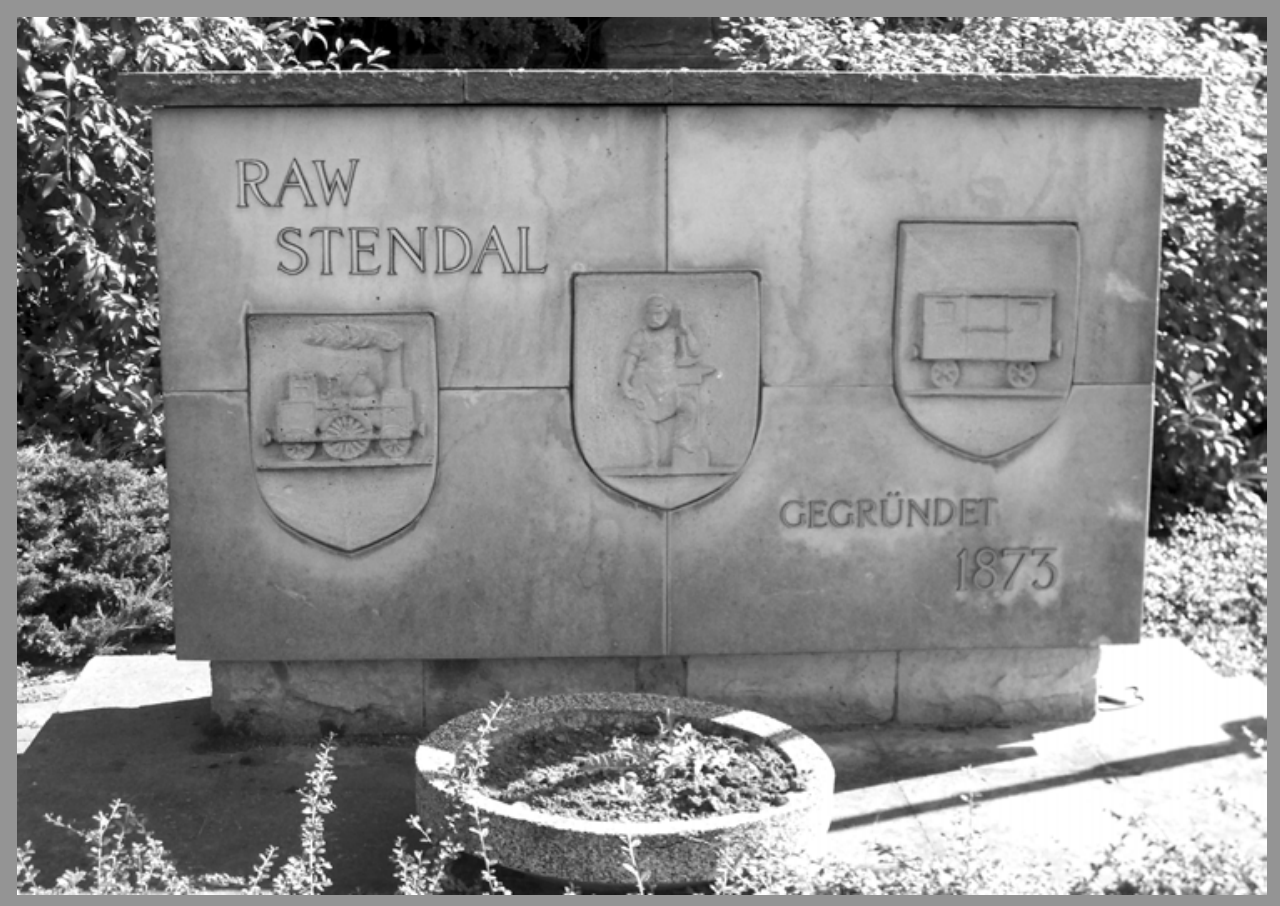

Aux ateliers ferroviaires de Stendal (Saxe), un forgeron entre locomotive et wagon à l'entrée du site. Cl. Christian Chevandier, août 2007. 
On n'imaginerait pas d'autres activités tertiaires présentées avec des hommes (et des femmes) si discrets. Pas d'enseignement sans professeur, de secrétariat sans secrétaire, de police sans policier, de commerce sans commerçant, d'activité postale sans postier. Le chemin de fer est différent. La technologie prévaut et la machine, sans le dissimuler tout à fait, escamote usuellement l'homme, tout comme ailleurs, dans l'asile de fous ${ }^{45}$ ou dans la prison, le bâtiment peut cacher ceux qui y pratiquent des activités coercitives. Y aurait-il une autre dimension qui tendrait à occulter le travailleur, celle de l'institution totale ? Dans cette hypothèse, celui des chemins de fer serait logiquement dissimulé. Sur l'image, sur ce qu'elle soumet d'un groupe ou d'un individu (y compris dans les portraits de groupe), sur ce qu'elle révèle d'un rapport à l'activité, sur ce que peut signifier son existence même, l'approche comparative est indispensable, à laquelle on soumettrait toutes les hypothèses, celles qui renverraient aux travaux des sociologues de la tradition de Chicago comme celles qui poseraient la question, toute simple, de la technique, de la technologie.

\section{La vue de I'autre: construction exogène $d$ 'une représentation}

Ce 23 août 2006, dans la grande cour de la préfecture de police de Paris, comme chaque année, une cérémonie rassemble de nombreux policiers parisiens. Le clou en est traditionnellement le discours du ministre de l'Intérieur ; cette année, il glorifie « les policiers [qui] ont amplifié le mouvement lancé cinq jours ${ }^{46}$ plus tôt par les cheminots ». Hors même du groupe social, les cheminots ont une image très forte, qui les présente comme exemplaires. Le même groupe social des policiers, tout comme celui des pompiers, agrémente depuis 1995 ses manifestations des fumigènes rouges qui ont contribué au succès esthétique des manifestations de cheminots ${ }^{47}$.

45- Sur un temps long, il semble que les photographies de pensionnaires d'hospices ou d'asiles départementaux sont plus communes que celles des patients d'hôpitaux. Mais les soignants de ces derniers établissements sont plus communément fixés sur la pellicule que les travailleurs d'hospices ou d'asiles. Ces premières impressions, qui demanderaient bien sûr à être approfondies, posent aussi la question du rapport à l'autre et, dans les activités de services, de l'influence de l'usager ou du client sur l'image et la représentation du travailleur.

46- Nicolas Sarkozy semble faire allusion ici non à l'insurrection du 19 mais à l'appel à la grève lancé le 15 août à l'appel du Front national de la police, de Police et Patrie et de Honneur de la police.

47- Voir ainsi la magnifique couverture en camaïeu rouge de Télérama, 13 décembre 1995. Des processus de localisation par satellite des trains en difficulté se substituent à ces signaux alors qu'ils acquièrent un statut symbolique. Ce décalage, qui évoque un usage jamais démenti des locomotives à vapeur parmi les allégories mises en œuvre par la corporation, mérite d'être interrogé. 
Hors du monde ferroviaire, la représentation des travailleurs du rail est, forcément, ambiguë. Nous pourrions multiplier les exemples parmi lesquels Le Chant des chemins de fer, une des rares commandes dans l'œuvre de Berlioz, à l'occasion de l'arrivée du chemin de fer à Lille (1846), qui, sur des paroles de Jules Janin, un des journalistes les plus célèbres de son temps, couvre de lauriers ses constructeurs ${ }^{48}$. Mais, même en milieu ouvrier, la carrière ferroviaire n'est pas toujours présentée comme une panacée et un père pouvait bien, dans l'entre-deux-guerres, menacer son fils : «Si tu réussis le concours des bourses, tu seras boursier [...]. Si tu ne réussis pas [...], tu iras à l'école des cheminots [...] pour conduire les trains. » Quitte, éventuellement, à ce que le gamin ne soit pas accablé par cette éventualitét9. Mais une telle complexité est bien signe d'un intérêt, loin d'une impossible indifférence. Parfois, cela va plus loin. Dans une émission fort sérieuse sur France Culture, lorsqu'il fut question de la grave dépression et de la célèbre mésaventure de ce président de la République, le journaliste s'est autorisé : « Paul Deschanel sait qu'il déraille». Hors d'une plaisanterie facile, nous savons à quel point le train fascine ceux qui veulent en finir, qui veulent le prendre pour un dernier voyage. Il y eut des suicides sur la première ligne, celle de Paris à Saint-Germain-en-Laye. Plus tard, pour son personnage d'Anna Karenine, Tolstoï s'est inspiré de faits réels, l'histoire d'une femme qui s'est placée sur des rails parce qu'elle avait été quittée par son amant. Quant au peintre lyonnais Louis Beysson, auteur d'un Voyage autour des voies ferrées et auquel nous devons des toiles où la gare de Perrache apparait sous des angles surprenant ${ }^{50}$, il a fini par se jeter un jour de l'été 1912 sous une locomotive ${ }^{51}$. Sigmund Freud, dans un article de 1920 à propos d'une de ses patientes qui s'était précipitée d'un

48- «C'est le grand jour/ Le jour de fête/ Jour des triomphes et des lauriers/ La couronne est prête/ Pour vous ouvriers/ Soldats de la paix/ C'est votre victoire/ C'est à vous la gloire/ De tant de bienfaits ». Il fut joué en novembre 1982 par l'Orchestre national de Lille, avec le ténor Alain Vanzo.

49- «Finalement, ça me plaisait de conduire les trains. Alors je me suis dit, si je ne réussis pas [...], eh bien, ma foi, je conduirai des trains et ça me disait quelque chose de conduire des trains, mais j’ai réussi le concours des bourses », Pierre Mauroy, « RadioSouvenir », France-Culture, 22 août 2006. Michel Onfray décrit en d'autres termes son orientation professionnelle : «Je me suis inscrit en philosophie parce que, à défaut de conduire des locomotives, je ne voyais pas quoi faire d'autre », Le Nouvel Observateur, 5 avril 2007.

50- Voir notamment La Gare au printemps et La Gare de Perrache, dans la collection permanente du musée des Beaux-Arts de Lyon.

51- Paysagistes lyonnais, Lyon, Musée des Beaux-Arts, 1984. 
pont de chemin de fer, n'a pas cru bon de souligner la dimension ferroviaire du geste ${ }^{52}$. En revanche, Alfred Hitchcock a lourdement insisté sur la dimension symbolique qu'il accorde à un train pénétrant dans un tunnel lors de la scène finale de North by Northwes $\tilde{p}^{3}$.

C'est que le public est sollicité de mille façons. Alors que certains groupes sociaux gardent pour eux les marques de leurs actions, au mieux les situent dans l'interface qui les sépare autant qu'elle les relie au reste de la population, c'est dans les espaces où passent et stationnent les voyageurs que les principaux monuments corporatifs aux morts ont été édifiés, c'est dans les gares que certaines manifestations (les expositions corporatives d'artistes amateurs notamment) se déroulent. Au risque, parfois d'une certaine incompréhension tant le langage du groupe social peut être hermétique ou prêter à confusion ${ }^{54}$. Et l'importance de l'engagement politique des cheminots ne les situe pas aux marges de la cité. «Les mineurs, les cheminots, les canuts » : avec « les congés payés, Le Temps des cerises, les enfants dans les mines ", avec "Jaurès et Clemenceau », les travailleurs du rail tiennent place dans « ce pot-pourri d'images et de symboles » qui construisent ce que le psychanalyste Jacques-Alain Miller caractérise comme « l'Homme-de-gauche ${ }^{55}$. Son approche est trop contemporaine et mériterait d'être élargie, notamment en prenant en compte les diverses élites du groupe social. Nous savons à ce propos quelle place les travailleurs de rail ont tenue parmi les leaders syndicaux, notamment à la CGT qui a puisé dans leurs rangs plusieurs secrétaires généraux, mais l'influence des ingénieurs a longtemps été considérable, même après la nationalisation. La figure de Raoul Dautry est notoire ${ }^{56}$. Il ne fut pas le seul. Charles de Gaulle ne se connaissait qu'un seul rival, Tintin, mais il aurait bien pu y substituer le Petit Prince, puisque Pierre Sudreau, modèle enfant du personnage de

52- Sigmund Freud, « Sur la psychogenèse d'un cas d'homosexualité féminine », Nérrose, psychose et perversion, Paris, Presses universitaires de France, 1973, p. 245-270.

53- 1959.

54- Ainsi, à la gare de Tergnier, grande ville cheminote du département de l'Aisne, une plaque en marbre rend hommage à Pierre Semard et précise qu'il fut «Administrateur Salarié de la SNCF ». Le cheminot comprend qu'il était représentant des travailleurs au conseil d'administration de la société nationale tandis que le passant béotien croit pouvoir supposer que l'expression désigne les membres d'une catégorie d'employés stipendiés.

55- Jacques-Alain Miller, «Tombeau de l'Homme-de-gauche », Le Monde, 4 décembre 2002. Remarquons que les canuts et les mineurs n'existent plus.

56- Rémi Baudoui, Raoul Dautry, 1881-1951. Le technocrate de la République, Paris, Balland, 1992. 
Saint-Exupéry, fut pressenti comme candidat contre le général aux élections de 1965, et se récusa. Tout comme Louis Armand. Mais si l'évocation pour ces premières présidentielles, de nouveau au suffrage universel, de ces deux hommes, au caractère très gaullien et dont le parcours passa par le gaullisme ${ }^{57}$, seules éventuelles candidatures sérieuses parmi ce que l'on n'appelait pas encore la société civile, peut surprendre, elle n'en ramène pas moins à cette identité cheminote qui irradie tous ceux qui, à un degré ou un autre, ont travaillé dans les chemins de fer.

La réputation des chemins de fer rejaillit sur ceux qui y travaillent. D'où cette surprenante culpabilité lorsque l'institution ou la technologie sont attaquées et que les cheminots se sentent visés. Le cas du grief qui leur a été fait d'avoir conduit les trains de la déportation a déjà été étudié. Le phénomène se produit également lorsque des agressions sont commises dans un train ou sur un site ferroviaire. Les questions de la fraude, certes anciennes ${ }^{58}$, et de la sécurité des voyageurs se posent, pas seulement en termes d'intégrité physique mais également parce qu'elles remettent en cause le lien social. Le caractère inopérant des contrôles et des amendes qui s'accumulent sans jamais être perçues ${ }^{59}$ se situe dans ce cadre. Quelle est la légitimité d'un contrôleur (la dénomination a changé, mais ils continuent à contrôler...) qui réclame un complément à un voyageur dont la date limite de la carte de réduction « familles nombreuses » est dépassée d'une semaine et qui n'a pas été renouvelée à temps par la société sous-traitante, lorsque le même usager peut voir certains de ses collègues s'abstenir de contrôler les justificatifs de règlement du trajet d'un petit groupe menaçant sans pour autant oublier les voyageurs plus

57- C'est moins évident pour Louis Armand, dont le gaullisme primitif, mélange d'honneur et de détermination, est proche de celui qui a pu caractériser des hommes aussi différents qu'André Philip ou Jacques Soustelle. Pour Pierre Sudreau, cette dimension est postérieure à l'engagement initial dans la Résistance, Pierre Sudreau, Au-delà de toutes les frontières, Paris, Odile Jacob, 1991. Voir également Christiane Rimbaud, Pierre Sudreau. Un homme libre, Paris, Le Cherche Midi, 2004, notamment le chapitre VII, «Ambassadeur du ferroviaire ».

58- Les voyageurs clandestins datent du début du chemin de fer et la pratique qui consistait à enfermer les voyageurs pour empêcher la fraude fut une des causes des 55 décès lors de l'accident de Meudon, en 1842. Aux États-Unis, la culture des hobos, les chemineaux locaux, s'est échafaudée autour de ces voyages illégaux à la dimension également initiatique, voir Jack London, La Route - Les vagabonds du rail, nouvelle traduction, Paris, Phébus, 2001.

59- La loi de 2002 n'a pas fondamentalement modifié ces pratiques qui mettent le voyageur mal à l'aise et déstabilisent les agents. 
paisibles? Un tel refus d'affirmer l'état de droit, refus qui au demeurant peut être le fait de l'institution elle-même ${ }^{60}$, contribue à dissoudre le lien social, remet en cause le bien-fondé de l'activité des agents en contact avec le public et atteint le groupe social dans son ensemble. Les agressions dont les voyageurs sont les victimes contribuent à entretenir une représentation négative du réseau ferroviaire qui concerne ses employés, souvent eux-mêmes victimes de violences. Bien plus graves, les conséquences des crimes commis sur le réseau ferroviaire se situent dans un autre registre que celui de l'Orient Express et d'Agatha Christie, avec des va-et-vient entre la fiction et la réalité. En 1984, le film Train d'enfers'inspirait largement du meurtre commis quelques années plus tôt sur le Paris-Vintimille. En décembre 2003, deux hommes étaient condamnés pour avoir jeté par la fenêtre d'un train, au moment où le convoi franchissait un viaduc qui enjambe la Seine, un voyageur qui refusait d'être racketté. En août 2001, une cheminote avait failli subir le même sort dans le train Marseille-Nice, celui-là même qui acquit en janvier 2006 une certaine notoriété à la suite des exactions dont furent victimes les voyageurs. Les meurtres commis en 1999 par Sid Ahmed Rezala, le « tueur des trains », sont caractéristiques du lien entre les fraudes et des agressions extrêmement violentes : cet homme, qui a tué deux voyageuses, était un habitué des trajets " sans billet », pour reprendre l'euphémisme d'usage. Dans ce contexte, la campagne publicitaire qui se voulait humoristique menée début 2006 par la SNCF peut pour le moins prêter à confusion. Présentant, parmi un corpus d'autres affiches, la photographie du quai désert d'une grande gare, un panneau y indique la gare de «Pétoches » pour rassurer l'usager craintif : «On n’est pas obligé de payer sur Internet. » Le contresens probable est malvenu alors qu'en 15 ans le nombre d'agents SNCF victimes d'atteintes physiques a presque triplé ${ }^{61}$, que les organisations syndicales dénoncent les réductions de personnel comme une des sources de l'insécurité, que nombre de grèves localisées ont eu lieu depuis vingt ans à la suite d'agressions et qu'il n'est pas inenvisageable qu'un mouvement social d'ampleur se développe sur ce thème.

60- Ainsi, au milieu des années 1990, constatant que certains trains ne sont empruntés que par des fraudeurs, la SNCF a mis en place une formule de «billet social » [sic] afin que les contrevenants puissent bénéficier de titres de transport à taux très réduit. La tentative ne fut pas couronnée de succès et un cadre de la SNCF insinua, pour plaisanter, que la société «gagnerait peut-être à offrir la gratuité aux jeunes fréquentant certaines lignes à risque, évitant ainsi des dépenses considérables en matière de contrôle et de lutte contre l'insécurité », Archives de la préfecture de police de Paris, dossier S23. 61- Entre 1990 et 2005, il est passé de 410 à 1 100, Thierry GerberR et alii, Violences contre agents. Agressions et souffrance dans les services publics, Paris, Gawsewitch, 2007, p. 157. 
Cette image des travailleurs des chemins de fer peut fort bien être construite hors même de l'action des cheminots. Leur participation à la Résistance, principal pilier aujourd'hui de la dimension héroïque de la réputation du groupe social, s'étaye encore par l'évocation du sabotage des voies ferrées, actions qui souvent s'accomplissaient sans le moindre recours aux cheminots. Le film Bataille du rail, dont l'intrigue est basée en partie sur la démonstration de l'efficacité de professionnels opposée à l'échec des amateurs d'un maquis, a joué son rôle dans cette construction. Mais la réputation vient de loin, des premiers sabotages réalisés dans le pays lors de la guerre de 1870 . Nous savons combien les armées d'Allemagne ont été impressionnées, tourmentées par l'activité des francs-tireurs lors de cette guerre, à quel point leur souvenir a pu jouer lors des conflits suivants. Elle fut pourtant bien limitée, il n'y eut pas vraiment d'attaque de convois et les quelques coups de mains et escarmouches n'eurent aucun impact militaire. Les principales réussites se limitèrent à quelques trains qui ont déraillé, des ponts qui ont sauté, des tunnels qui se sont écroulés. Associé à ces actions, le train entraîna dans sa réputation, sans qu'ils n'y fussent pour rien, les travailleurs des compagnies ferroviaires. Mais ne nous y trompons pas, le regard porté sur les représentations d'une résistance ferroviaire ne rend pas, bien sûr, invariablement ferroviaire toute résistance, pas plus que toute allusion au rôle politique du chemin de fer ne renvoie systématiquement à la Résistance : dans Didascalie se promenant seule dans un théatre vide, Armand Gatti introduit le chemin de fer pour évoquer la révolution soviétique et la déportation vers Auschwitz ${ }^{62}$, mais pas de l'action d'une Résistance omniprésente dans sa pièce.

Une étude systématique de la place des cheminots dans le cinéma nous montrerait peut-être qu'il s'agit d'un groupe insoumis et résolu, de ceux de Dino Risi qui foncent sur le véhicule des chemises noires placé sur un passage à niveau ${ }^{63}$ à celui de Ken Loach qui choisit d'être tabassé par les soldats anglais pour ne pas contrevenir aux consignes du syndicat ${ }^{64}$. Mais le cas français est, par sa genèse, particulier. Un peu comme il a su construire sa propre image cinématographique, en se figurant dans Bataille du rail de René Clément, dans Nadia et les hippopotames de Dominique Cabrera, le groupe social des travailleurs des chemins de fer finit souvent par s'approprier les représentations, même lorsqu'il n'y est pas à la fête. Le Lantier joué par Gabin est devenu un

62- « Les quais de gare répondront aux quais de gare notre voyage durant. »

63- La Marcia su Roma, 1962.

64- The Wind That Shakes the Barley, 2006. 
personnage essentiel, au sens premier du mot, aussi surprenant que cela soit pour un meurtrier psychopathe. Une des premières scènes du film permet d'identifier le mécanicien à la machine ${ }^{65}$, lorsqu'il fait un geste et semble demander à boire alors que c'est la locomotive qui a besoin d'eau. Cet accueil tranche avec celui réservé au film d'Abel Gance à sa sortie : « Nous n'avons pas vu La Roue, mais des échos nous viennent qui nous la présentent comme une caricature de notre corporation, comme un danger social » écrivait Pierre Semard ${ }^{66}$. Pourtant, déjà, des cheminots avaient été sollicités pour y jouer leur propre rôle. Sans doute la dimension politique et collective, qui n'empêche pas l'inscription de la singularité individuelle dans les films de Clément et de Cabrera ${ }^{67}$, explique-t-elle ce sort différent. Mais c'est à l'occasion du $70^{\mathrm{e}}$ anniversaire de la SNCF que le Comité d'établissement régional cheminots Nord - Pas-de-Calais organise une soirée ${ }^{68}$ pour la sortie de l'ouvrage de Frédéric H. Fajardie, Tu ressembles à ma mor ${ }^{69}$, qui relate l'enquête policière qui suit, aux lendemains de la nationalisation, le meurtre de trois cheminots.

Comme dans tous les domaines ${ }^{70}$, une approche comparative n'en est pas moins nécessaire, qui peut se faire par plusieurs biais. La représentation du groupe social des cheminots introduit en France nombre de spécificités, sur lesquelles il conviendrait de s'attarder : voir comment, ici ou ailleurs, elle s'inscrit dans une vision plus globale, celle du prolétariat, et quel est le rapport entre les groupes qui manipulent les symboles, chercheurs et journalistes, et les milieux populaires. Le mépris

65- Il est même possible d'identifier la corporation à la machine (selon le contresens classique qui désigne la locomotive par l'expression qui est censée caractériser Lantier, prisonnier de son hérédité et de sa maladie mentale) : «Gallois n'est pas dupe. Si la droite l'a nommé, c'est que la SNCF est une poudrière et que mieux vaut un patron de gauche pour chevaucher la bête humaine », Libération, 17 mai 2006.

66- La version définitive du film fut amputée de nombreuses scènes, Michel Ionascu, op. cit., p. 249.

67- Philippe Corcuff, «La singularité individuelle en «jeux de langage». Sur quelques interférences entre la philosophie de Jacques Rancière, la sociologie de Pierre Bourdieu et le film Nadia et les hippopotames», Transversale-Arts en sciences en recherche, $\mathrm{n}^{\circ} 1$ (2005), p. 200-206.

68- Le 13 avril 2007; à cette occasion, on y projette La Bête humaine.

69- Édition des Équateurs, 2007.

70- Voir ainsi, pour une analyse de la pratique de la lecture chez les cheminots et les papetiers, à partir notamment de sources orales, Nathalie Ponsard, Lectures ouvrières à Saint-Etienne-du-Rourray, des années trente aux années quatre-vingt-dix, Paris l'Harmattan, 2007. La démarche comparative est d'autant plus féconde que l'auteur mène ses analyses à différentes échelles, notamment celle de l'individu. 
dont sont les victimes les cheminots anglais et leur réputation de balourdise, qu'au demeurant certains assument volontiers, relèvent des structures d'une société extrêmement clivée, où le parcours de Richard Hoggart est exceptionnel alors que les scientifiques français issus de milieux populaires ne sont pas rares. En France, la popularité de ses travailleurs renvoie également à celle de ce moyen de transport dans la classe ouvrière, associé aux congés payés, donc au Front populaire ${ }^{71}$. Sans doute, la place des cheminots français s'inscrit-elle dans l'histoire du pays, dans celle du peuple de France et est en cela bien particulière ${ }^{72}$, expliquant le caractère ouvert du groupe et sa tradition d'accueil, de solidarité. Leur réputation d'héroissme, antérieure à la période de la Résistance, devrait aussi être interrogée ainsi. Ce n'est sans doute pas par hasard si c'est aux États-Unis d'Amérique, dans le pays où a été posée pour la première fois, au début des années 1980, la question du rôle des cheminots français dans les déportations lors de la Seconde Guerre mondiale $^{73}$, qu'une réponse y avait été apportée plus d'une quinzaine d'années plus tôt : «Qu'on l'arrête avec nos mains ? Qu'on se couche en travers des rails ${ }^{74}$ ?»

71- Collection Roger-Viollet, Les Congés payés en photos, Paris, Hachette, « Ruée vers les gares », p. 34-49. Sur un seul de ces clichés, celui des chargements de bicyclettes gare Saint-Lazare en juillet 1936 (p. 38-39), il est possible de distinguer des cheminots. Plus largement, les gares ont été le lieu de nombre de manifestations populaires, lieux d'accueil pour le retour des combattants des Brigades internationales, des prisonniers, des déportés. Cette dimension symbolique explique notamment que la gare de Lyon fut un enjeu (de même nature que la manifestation à Billancourt du 16) le 24 mai 1968, et c'est dans son prolongement qu'il faut appréhender le débat qui s'y tint, animé par Pierre Bourdieu, le 12 décembre 1995. Voir l'analyse par Pamela E. Swett des gares du S-Bahn de Berlin comme lieu politique : «Political Networks, Rail Networks: Public Transportation and Neighbourhood Radicalism in Weimar Berlin ", in Ralf Roth et M.-N. Polino (dir.), The City and the Railway in Europe, Aldershot, Ashgate, 2003, p. 221-235, et sa thèse: Neighbors and Enemies: The Culture of Radicalism in Berlin 19291933, Cambridge, Cambridge University Press, 2004, xvi-337 p.

72- Il n'y a rien sur les chemins de fer dans les deux ouvrages de Richard Hoggart, $L a$ Culture du paurre, Paris, Éditions de Minuit, 1970, et 33 Newport Street, Paris, Seuil, 1991.

73- Il ne s'agit bien sûr que de la dimension intellectuelle et civique de cette question, voir Christian Chevandier, "Le grief fait aux cheminots d'avoir, sous l'Occupation, conduit les trains de la déportation", in "Les cheminots dans la Résistance. Une histoire en évolution", Actes du colloque du 3 décembre 2005, Revue d'histoire des chemins de fer, $\mathrm{n}^{\circ} 34$ (printemps 2006), p. 91-111. L'effet du mode de rémunération des avocats aux États-Unis n'est intervenu que dans un second temps.

74- John Frankenheimer, Le Train (1964). Il s'agissait, dans ce film dont le scénario était tiré du roman de Rose Valland, Le Front de l'art, d'empêcher un train d'œuvres d'art de partir pour le Reich au moment de la Libération de Paris. 


\section{La vue de soi : construction endogène d'une identité}

Antoine B. est né quelques temps avant la Grande Guerre. Un lundi matin sans doute, alors qu'il avait une vingtaine d'années, il s'est fait photographier avec un copain un peu plus âgé devant la cabine d'une locomotive. Antoine B. a dans la main un chiffon, comme s'il venait de lustrer la machine, et l'ainé a posé la main sur son épaule. La photo est archétypique de celle d'une équipe de conduite, mais les deux hommes travaillent au Montage de l'Atelier d'Oullins-Machines. Antoine B. est ajusteur, gardera la photographie dans son portefeuille pendant des décennies et lorsqu'on lui demande s'il a voulu un jour conduire des locomotives, il se récrie : «Jamais! ! Il est des images qui sont un discours plus fort que les discours.

La vue de soi, c'est aussi l'écriture de soi. Aucun travail de fond n'a jusqu'à présent été accompli sur les autobiographies de cheminots. L'analyse de tels documents, aujourd'hui, a été largement accomplie et se révèle classique. Les groupes qui ont écrit sont, il n'y a rien là de surprenant, ceux qui maitrisent l'écriture. Les textes d'ingénieurs ou de cadres administratifs ${ }^{75}$ sont dès lors plus courants que ceux des travailleurs moins bien placés dans la hiérarchie ferroviaire, moins à l'aise dans la pratique de l'écriture. Les rares autobiographies de cheminots dont nous disposons sont communément convenues et répétitives, comme ce texte de Marcel Péroche que nous citons à l'envi ${ }^{76}$. Ils peuvent également être pesamment démonstratifs, libelles militants qui se veulent prosélytes et ressassent toujours les mêmes justifications ${ }^{77}$. On

75- Voir ainsi les textes autobiographiques de Frédéric Surleau et Robert Lévi, « Mémoires d'ingénieurs, destins ferroviaires. Autobiographies professionnelles de Frédéric Surleau (1884-1972) et Robert Lévi (1895-1981) », Revue d'histoire des chemins de fer hors série, $\mathrm{n}^{\circ} 8,2007$.

76- Notamment Marcel Péroche, Les Mémoires de Marcel Péroche, "sénateur» du rail, Paris, Berger-Levrault, 1984. Une traduction en anglais en a été éditée récemment, Pacific Senator: A Train Drivers Life, Glendaruel, Argyll Publishing, 2005, traduction et introduction par Roland Wilson. Voir également Ne touche pas à ma locomotive! Scènes de la vie de Marcel Péroche, ancien mécanicien de l'Orient Express, Paris, France-Empire, 1990, propos recueillis par André Fonnet. Les ouvrages d'Henri Vincenot ne relèvent pas de cette catégorie d'autobiographie cheminote.

77- C'est ce que l'on peut également trouver dans certaines biographies. Voir ainsi le passage consacré à son père dans la notice de Joseph Jacquet écrite par Maurice Moissonnier : « Il avait participé aux grandes grèves de l'immédiat après-guerre, tout en révélant un tempérament libertaire par des comportements qui s'éloignaient parfois de la discipline de lutte », Marie-Louise Goergen (dir.), op. cit., p. 252-255. Il nous explique également, en un succulent euphémisme, que Joseph Jacquet « ne comprit pas les raisons du pacte germano-soviétique ». 
pourrait être tenté d'aller chercher du côté de la fiction pour mieux appréhender ce groupe social, et c'est ainsi que le roman anticolonialiste qu'a écrit en prison Pierre Semard complète, dans le sens d'une appréhension de l'homme, ses textes militants ${ }^{78}$. Il n'en reste pas moins que si une typologie de l'écriture devait être esquissée, les cheminots y apparaitraient moins prolifiques qu'un groupe social proche, tel celui des postiers $^{79}$. Ce n'est que lors du dernier quart du vingtième siècle, dans le contexte d'un accroissement considérable du niveau scolaire de la population française et en une dynamique que l'on retrouve dans les autres groupes sociaux, que la donne a changé $e^{80}$ et que ces textes sont devenus plus nombreux, leur écriture et leur publication ayant été également stimulées par les grandes grèves de 1986 et $1995^{81}$, alors que les cheminots ne se trouvaient pas parmi les auteurs d'autobiographies publiées dans les années $1970^{82}$. Il est probable que l'outil informatique, soulageant l'écriture de soi de ce qui la rendait malaisée en milieu populaire, permettra la multiplication de tels textes ${ }^{83}$. La question se posera alors de l'accès à ces documents et de la conservation des archives numériques. Mais nous n'en sommes pas là et une étude du phénomène autobiographique dans le monde des travailleurs des chemins de fer se

78- Serge Wolikow, «Un roman anticolonialiste : «Bamba », le petit diable noir de Pierre Semard ", in Sophie Beroud, Tania Regin (coord.), Le Roman social, littérature, histoire et mouvement ouvrier, Paris, Éditions de l'Atelier, 2002, p. 160-166.

79- Un rapide inventaire des romans postiers (avec 8 titres, écrits par des postiers des grades inférieurs) révèle une différence de taille avec les romans cheminots (5 titres, avec comme auteurs Paul Nizan, Pierre Hamp, Emile Zola...). Le type de sélection à l'embauche tout comme le rapport à l'écrit particulier chez les travailleurs des postes expliquent en partie cette différence.

80- C'est en partie ce qui explique la qualité de l'autobiographie de Bernard Thibault, d'une grande richesse et à mille lieues des écrits militants antérieurs, Ma voie ourrière, Paris, Stock, 2005. Le jeu de mots qui constitue le titre, parvenant à associer la classe, la corporation et la tradition syndicaliste française est à cet égard significatif de l'ouverture caractéristique du groupe social des travailleurs des chemins de fer.

81- Voir par exemple Pierre Blairet, Cheminot, Paris, Éditions du Rocher, 1998 (dans la collection « Ma vie est mon métier», dirigée par Alphonse Boudart), et Roger Habert, Conducteur de locomotive : une vie pour l'honneur du rail, Paris, L'Harmattan, 1999.

82- Notons l'absence de Moi, un cheminot dans la collection des éditions Stock de ces années-là.

83- Philippe Lejeune, "Cher écran... » Journal personnel, ordinateur, Internet, Paris, Seuil, 2000 ; l'ouvrage de base reste celui qu'il a publié chez le même éditeur en 1975, Le Pacte autobiographique. 
révèle d'autant plus indispensable qu'elle permettrait une autre approche de l'important corpus de sources orales en cours de constitution et de collecte ${ }^{84}$.

Ce n'est que depuis la fin du XIX siècle que les cheminots sont des cheminots. Auparavant, le mot n'existait pas. Il a, depuis, joué un rôle énorme ${ }^{85}$, unifiant le groupe alors que son éclatement est perçu comme un grand danger ${ }^{86}$. Pourtant, alors que l'on sait à quel point c'est par un rapport dialectique que l'image construit l'identité, il est nécessaire de prendre la mesure de la place des cheminots atypiques, ceux qui seraient écartés dans la construction d'un idéal-type. C'est le cas de ces "petits cheminots", tous ceux qui ne sont ni dans les six grandes compagnies ${ }^{87}$, ni ensuite à la $\mathrm{SNCF}$, et c'est précisément un coup de zoom sur cette périphérie qui peut nous en apprendre sur l'ensemble. Ces cheminots atypiques seraient de mille ordres. Il y aurait les non-grévistes, non ceux qui sont instrumentalisés par la direction de la SNCF pour une opération médiatique lors de la grève de 1986-198788

84- « Pour un programme de collecte d'archives orales : le personnel de la SNCF et son entreprise, 1937-2002. Histoire professionnelle d'un corps social, évolutions et représentations ", Dossier, Revue d'histoire des chemins de fer, $\mathrm{n}^{\circ} 31$ (automne 2004), p. 5-76.

85- Les enjeux ne sont pas minces, qui expliquent les batailles taxinomiques comme celle qu'a tentée (en vain) la direction de la SNCF pour imposer le syntagme "pilote » aux conducteurs de TGV, alors que l'usage est encore commun du mot «mécanicien » qui désignait à l'origine les conducteurs de toutes les machines à vapeur, y compris fixes. En 1955, l'INSEE distinguait bien les conducteurs, pour les locomotives électriques, des mécaniciens, pour les locomotives à vapeur, Commission interministérielle de la nomenclature des métiers, Dictionnaire des métiers et appellations d'emploi, Paris, Presses universitaires de France. Notons à ce propos qu’à la métaphore habituelle de la « locomotive de la grève ", Pierre-Marie Thiaville et Marcel Trillat (qui, il est vrai, n'appartiennent pas à la corporation) ont préféré, pour présenter Bernard Thibault, " [il] a piloté en 1995 l'une des plus grandes grèves de la SNCF », Bernard Thibault, op. cit., p. 7.

86- C'est à cette aune qu'il faut comprendre les réactions qui pourraient sembler excessives lorsque certains travaux d'historiens insistent sur la diversité des comportements, par exemple en réfléchissant à une chronologie des mouvements sociaux dans les chemins de fer en fonction de l'implication des différents services.

87- Ou dans un réseau de l'État lorsque certaines d'entre elles se révélèrent défaillantes.

88- La centrale d'achat de la SNCF a fait publier dans Le Monde après presque trois semaines de grève un communiqué anonyme de cadres de la direction commerciale voyageurs de Paris-Saint-Lazare attaquant violemment les grévistes, rapports des Renseignements généraux, 7 janvier et 26 février 1987, Archives de la préfecture de police de Paris, dossier S24. 
mais tous ceux qui souffrent réellement lorsque leurs compagnons cessent le travail parce qu'ils ont l'impression que les grévistes salissent le monde ferroviaire. Il y aurait aussi, dans un groupe social perçu (à juste titre) comme résistant, les collaborateurs, ces rares cheminots qui ont milité au PPF ou au RNP, ceux qui ont fini dans la Milice ${ }^{89}$.

Ce changement d'échelle, de focale, d'objet, la multiplicité des biais sont des outils dont ce volume nous montre l'efficience. La division au sein du monde cheminot entre les métiers, les services, les sites même, est si redoutée de la corporation que sa réalité en est, dans les représentations, hypertrophiée, à tel point que cela risquerait de nous faire oublier qu'elle est moindre que dans bien des secteurs. Le monde enseignant, ainsi, est stratifié en une multitude de hiérarchies édifiées sur des certitudes aussi précaires qu'assénées telles des évidences, comme si ce groupe professionnel cherchait dans des éléments marginaux sa raison d'être. Il est tentant de multiplier les exemples comme celui de l'expérience de George Orwell, à la plonge dans un restaurant parisien dans l'entre-deux-guerres, relatant que «cela ne se fait pas, pour un garçon, d'être affable avec un plongeur $»^{90}$. Le monde des cheminots est plus unifié, plus uni que la plupart des milieux professionnels, mais il n'en est pas moins soumis à nombre d'éclatements, tel celui des générations. Rien ici de nouveau, rien d'original, le lieu du travail est celui d'initiations et de concurrences où se révèlent des antagonismes. Alors que le bizutage est moins marquant, moins traumatisant ici qu'ailleurs (mais il est rare que l'on commence jeune une carrière dans les chemins de fer, sauf si l'on est passé par un centre d'apprentissage où l'initiation est déjà accomplie), les rivalités n'en existent pas moins, on reste entre gens de son âge et seul le militantisme, syndical ou politique, parvient d'autant plus aisément à transcender ces différences que la voie de l'apprentissage dans des centres de la SNCF permet une intégration professionnelle et sociale qui concerne également, par une osmose générationnelle, les travailleurs qui, plus nombreux, deviennent cheminots après un parcours de mobilité d'entreprises ${ }^{91}$. Au-delà de cet éclatement par âges, il y aussi les dissimilitudes qui caractérisent des

89- Avant d'être proxénète et tortionnaire, Paul Touvier a été employé comme expéditionnaire dans les chemins de fer.

90- George Orwell, Down and out in Paris and London, Londres, Martin Secker et Warburg Limited, 1981 [1 $1^{\text {te }}$ édition, 1933], p. 46.

91- Pour la période la plus récente, voir Bernard Thibault, op. cit., p. 33-34. Une histoire de l'apprentissage dans les chemins de fer français, qui a été inégalement traité dans les approches monographiques, reste à écrire. 
générations. Les générations du feu, celle de la Grande Guerre (considérablement renforcée dans les chemins de fer par les recrutements de la journée de 8 heures, en 1919), celle de la Résistance, celle de la guerre d'Algérie, dont les membres partageaient la fierté ou les traumatismes, les jeunes recrutés dans l'après-soixante-huit et qui ont bouleversé le climat social dans la vieille entreprise, tous ces itinéraires différents et divergents ont joué pour fractionner le groupe social, et cela n'a rien de spécifiquement cheminot. Du même phénomène peuvent relever des différences de comportement caractérisées par la consommation de produits psychotropes : ce ne furent pas les mêmes générations qui ont bu du vin, du pastis, de la bière, consommé des dérivés du cannabis ${ }^{92}$. Même l'affirmation de tempérance peut aussi jouer pour individualiser, lorsqu'elle est perçue comme méprisante par certains, en des jeux d'individus et de groupes où l'âge et les générations se révèlent fondamentaux. Car si le cheminot archétypique n'existe pas, un idéal-type prendrait un homme, et un homme d'âge mûr, pas trop au demeurant puisqu'il s'agit d'un milieu où la retraite vient tôt.

Le monde des travailleurs du rail s'affiche aussi, et en cela les uniformes ${ }^{93}$ jouent leur rôle, souvent difficiles à interpréter (Que signifient toutes ces étoiles ? Est-ce là un contrôleur de la SNCF où un général de division ?), en un hermétisme qui distingue. Cette distinction s'affirme dans l'espace mais demeure trop discrète pour être vraiment opérante hors du groupe. Il s'agit de signes infimes, d'objets fabriqués en perruque et que l'on affiche dans la ville, invisibles à tout étranger à la corporation. Même les babioles confectionnées pour montrer le chemin de fer, petites silhouettes de locomotive à vapeur en fonte de 7 centimètres sur 14 sorties dans les années 1990 d'un Atelier SNCF, sont offertes à des étrangers à la corporation et en cela n'écartent pas mais impliquent. Et si des inventaires en ont été dressés, il y aurait une véritable étude à effectuer de l'usage et de la fonction de ces infimes insignes, petits badges, porte-clefs offerts lorsqu'un train bat un record

92- Ce qui explique notamment des changements dans les circonstances de certains accidents du travail : le malheur qui survient construit également une identité professionnelle, notamment dans des secteurs comme les chemins de fer où la culture de la sécurité est si forte.

93- L'origine militaire des uniformes n'est pas une spécificité française et relève plutôt de l'organisation sociale des sociétés au temps du développement des chemins de fer. Les uniformes de la Reichsbahn puis de la Bundesbahn sont d'abord des uniformes de travailleurs du rail, qui n'apparaissent comme militaires que parce qu'ils se sont moins civilisés depuis le XIX siècle que ceux de leurs collègues français. 
de vitesse, minuscules médailles remises par la SNCF pour célébrer l'événement et que j'ai vues portées par des syndicalistes, à côté de badge de leur organisation syndicale, lors d'une des grandes grèves de la fin du $\mathrm{XX}^{\mathrm{e}}$ siècle, insignes récupérés par les grévistes comme les fumigènes ou les poses à la Gabin, béret et lunettes de conduite arborés dans les cortèges.

Pour se distinguer, le cheminot ne se contente pas de montrer, il discerne :

«Sur la ligne, on voit tout, on connait tout.

- Avec cette vitesse ?

- Bien sûr! Ben, tenez. On suit très bien les saisons, avec les fruits qui poussent aux arbres, et puis qui grandissent, et puis qui tombent. Mais dans les champs, les p'tits lapins, vous savez, on voit, avec leurs petites oreilles là qui pointent dans les friches. Ils nous regardent passer. Ils n'ont pas peur. Ils savent très bien qu'on ne leur fera pas de mal.

- C'est drôle. Nous, quand on voyage et qu'on regarde par les portières, on ne voit rien de tout ça.»

Dans cette scène du film de Renoir, quand Lantier dit « on » ou "nous », il désigne le couple qu'il forme avec la Lison, sa locomotive ${ }^{94}$. Beaucoup a été dit sur les rapports entretenus par les conducteurs avec leur machine, et pourtant il convient de revenir sur cet élément, essentiel, tant il pourrait être somme toute révélateur d'un dysfonctionnement psychique. C'est bien de machines qu'il s'agit, d'objets inanimés, non d'humains ou même d'animaux ${ }^{95}$, et cet anthropomorphisme admis sans plus débats peut s'inscrire dans un ensemble de perceptions qui,

94- Emile Zola insiste sur l'ambiguité des rapports entre l'homme et la machine : « Elle portait le nom d'une gare, celui de Lison, une station du Cotentin. Mais Jacques, par tendresse, en avait fait un nom de femme, la Lison, comme il disait, avec une douceur caressante. Et, c'était vrai, il l'aimait d'amour, sa machine, depuis quatre ans qu'il la conduisait. [...] S'il l'aimait celle-là, c'était en vérité qu'elle avait des qualités rares de brave femme. »

95- Le petit texte où un résistant s'adresse en s'excusant à sa locomotive qu'il va saboter et en terminant par " Je ne suis pas un assassin! " (Bulletin des chemins de fer, $\mathbf{n}^{\circ}$ 2, novembre 1943, reproduit dans "Les cheminots dans la Résistance", La Lettre de la Fondation de la Résistance, $\mathrm{n}^{\circ}$ spécial, 2005, p. 12) est communément mis en avant comme significatif du déchirement de l'auteur de sabotages qui pouvaient faire de vraies victimes en chair et en os; c'est bien l'oubli de ces dernières qui devrait ici surprendre, une omission qui s'explique peut-être précisément parce que, pour les résistants, il était impensable de s'en prendre à des innocents. 
pour le coup, construiraient une spécificité. Là aussi, l'anthropologie religieuse permettrait de relire le texte de Renoir, qui n'est pas vraiment celui de Zola, et montrerait un cheminot initié qui voit ce que ne voit pas le voyageur profane ${ }^{96}$ : la question du regard (le regard qui distingue, mais également celui qui n'y parvient pas) est essentielle en histoire des représentations.

Lorsque nous avons organisé dans le cadre du séminaire la projection de Nadia et les hippopotames, j'ai eu une discussion avec Ariane Ascaride qui m’a expliqué pourquoi les autres comédiens ne seraient pas là : le film terminé, ils sont sollicités lors de sa sortie mais il faut ensuite qu'ils tournent la page, passent à autre chose, admettent que c'est terminé. Et comme je lui parlais des rapports, lors du tournage, entre les cheminots figurants, jouant leur histoire, et les comédiens : "Ah mais tu sais, c'est un métier tellement prenant, les cheminots qui ont joué leur propre rôle, ils ont été pris par ce métier ${ }^{97}$. » Moi qui imaginais les comédiens phagocytés par les cheminots, qui avais envisagé ce tournage comme l'immersion du monde du cinéma dans celui des chemins de fer, je passais à côté d'une autre dimension, celle sinon de la confrontation du moins des relations entre plusieurs métiers.

Ce texte indique des pistes, propose des itinéraires, pose nombre de questions, mais ces explorations ne sont possibles que parce que des domaines ont été reconnus, des inventaires effectués, des réponses apportées. Le bilan du séminaire puis du colloque est solide, travail issu de questionnements qui ont structuré nos approches sur une corporation exceptionnelle. C'est que rares sont les groupes sociaux ou professionnels si attentifs, si sensibles à leur image. L'enjeu, il est vrai, n'est pas mince : il s'agit simplement de construire en permanence une

96- Plus globalement, un grand colloque serait à faire sur le thème des chemins de fer et de la religion. Si l'on connait la place du chemin de fer dans l'imaginaire et dans l'œuvre des saint-simoniens, il ne s'inscrivit pas véritablement dans leurs considérations religieuses, Georges Ribeill, « Les chemins de fer : de la doctrine aux réalisations », in Nathalie Coilly et Philippe Regnier, Le Siècle des saint-simoniens du Nouveau christianisme au canal de Suez, Paris, Bibliothèque nationale de France, 2006, p. 131-135.

97- «Je ne suis pas sûre que des logiques professionnelles se soient confrontées. [...] Ils jouaient pour eux-mêmes, ça leur donnait du plaisir. Parfois c'était dur parce que c'était des choses qu'ils ont vécues. ", Entretien en juin 2005. Pour Ariane Ascaride comme pour le général de Gaulle, la citation écrite, loin de rendre le ton de la phrase, est toujours un peu frustrante. 
identité, de la préserver continuellement. L'image des cheminots est étayée par les images des cheminots. Mais ils ne se précipitent pas pour être sur la photographie, un peu comme si le cheminot argentique n'apparaissait que mal derrière la technique. Car s'il s'agit d'une identité fortement masculine, elle n'en reste pas moins basée sur la fierté de la technologie, du métier, sur un collectif conçu plus encore que perçu. Ce rapport à la technologie, à l'activité, au groupe, est si intense que l'on ne sait pas toujours, en dépit de l'évidence, ce dont il s'agit. En témoigne cette incertitude spontanée sur la classification (pourtant patente) de l'exercice ferroviaire parmi les secteurs dans la typologie de Colin Clark $^{98}$, comme si les Ateliers tiraient vers le secondaire une activité tertiaire, comme si la machine édifiait fallacieusement une transformation bien imaginaire. Par cela, le groupe social des cheminots est bien différent d'autres groupes professionnels.

Les résultats de nos études sur les images et les représentations nous amènent là où, déjà, les recherches sur les mobilités sociales ou d'autres travaux débouchant sur les sociabilités ${ }^{99}$ nous avaient portés : ce groupe social des travailleurs des chemins de fer est extraverti. Ils ne font pas partie d'un groupe à part, un groupe qui se coupe des autres, qui ne vit que dans l'entre soi. Les moments de gloire du passé, les grandes grèves et la Résistance, ne se sont pas voulus coupés de la société, de la nation, mais ont bien pris en compte l'intérêt de l'ensemble de la population, d'où ce caractère exemplaire, pédagogique, que les cheminots se plaisent à mettre en avant. À mettre en avant par des images, tout en étant sensibles à leur image, construction sans cesse en cours d'une représentation qui en est d'autant essentielle. D'où cette sensibilité à ce qui se dit, se pense sur la corporation. Les cheminots sont bien dans la société, dans le siècle, oserait-on écrire.

Il était question quelques lignes plus haut de profanes. Le monde des chemins de fer est délimité par des discernements, de mots et de sigles insolites (pensons aux chiffres et aux lettres qui désignent les locomotives), qui autorisent une interface moins clivante que ne le consentirait le seul critère de l'emploi par une institution ferroviaire. Nombre de cheminots qui ne sont pas conducteurs, peut-être même des chercheurs en sciences sociales ayant eu l'occasion de se pencher sur

98- «Les services. Définitions, ruptures, enjeux », Le Mowvement social, $\mathrm{n}^{\circ} 211$ (avriljuin 2005).

99- Christian Chevandier, "Construction identitaire et reconstruction: Sancy et les cheminots ", in Nicolas Offenstadt (dir.), Le Chemin des Dames. De l'événement à la mémoire, Paris, Stock, 2004, p. 382-392. 
le monde des travailleurs du rail, ont eu l'occasion de conduire des locomotives, sous l'autorité du conducteur en titre mais en pleine illégalité ${ }^{100}$. Ce rapport spécialiste/profane, patent dans des images comme la photographie d'Antoine B. dont il a été question supra, ne peut qu' inciter à s'intéresser aux travaux du sociologue américain Everett C. Hughes ${ }^{101}$, tout comme le rapport entre les métiers (le chauffeur dont la fonction consiste également à protéger le mécanicien, fonction perceptible dans nombre de représentations qui contribuent à renforcer cette dimension), l'office du "sale boulot ", la délégation des tâches, tous ces aspects apparaissent dans les images et nul doute qu'une approche théorique englobant notamment des références à cette génération des sociologues de Chicago pourrait être féconde. Car le rapport de l'individu au collectif est étayé dans les chemins de fer par bien des particularités, qui se révèlent fort prégnantes ${ }^{102}$. Le «nous» des cheminots $^{103}$, quand bien même il est éclaté, exprime bien une communauté de destin, un destin qui s'accomplit étayé par le passé, d'où cette attention, inhabituelle à ce point, portée à l'histoire du groupe : «À quoi ça sert d'avoir une histoire si on la ramène pas ${ }^{104}$ ? » Une attention qui passe par une sensibilité extrême aux images et aux représentations, à leur réception comme à leur construction.

100- Le récit en est souvent un moment fort lors du recueil de sources orales auprès de retraités d'autres services des chemins de fer que de celui de la Traction.

101- Everett C. Hughes, Le Regard sociologique. Essais choisis, textes rassemblés et présentés par Jean-Michel Chapoulie, Paris, Editions de l'EHESS, 2004.

102- C'est précisément la photographie de deux acteurs du film de Jean Renoir, Jean Gabin et Julien Carette, qui a été choisie par l'éditeur pour la couverture du tome second de l'Histoire des Chemins de fer en France (1883-1937) de François Caron (Paris, Fayard, 2005).

103- Cette identité collective qui n'a pas été laminée, au moment où un individualisme, qui est le produit des méthodes de gestion du personnel fait de véritables ravages dans le monde du travail (Christophe Dejours, Souffrance en France. La banalisation de l'injustice sociale, Paris, Le Seuil, 1998), contribue à renforcer dans ce domaine la spécificité du groupe social des cheminots. La question se pose néanmoins, parmi les facteurs de cette pérennité, de la place des représentations et des images.

104- Remarque d'un cheminot lors du tournage de la dernière séquence de Nadia et les hippopotames. 
